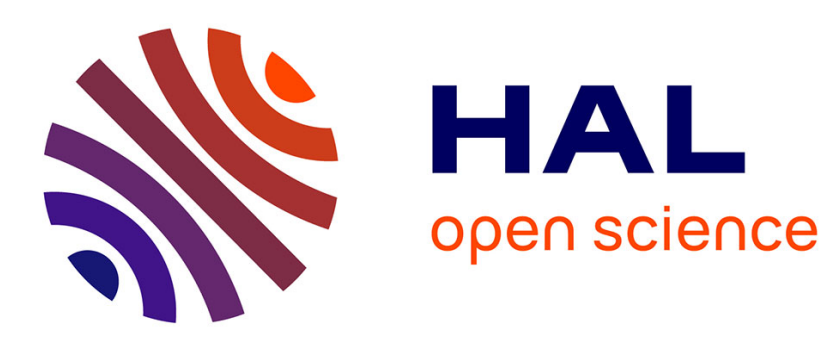

\title{
Some possible effects modifying the far infrared synchrotron radiation spectrum
}

R. Coisson

\section{To cite this version:}

R. Coisson. Some possible effects modifying the far infrared synchrotron radiation spectrum. Journal de Physique Lettres, 1984, 45 (2), pp.89-94. 10.1051/jphyslet:0198400450208900 . jpa-00232313

\section{HAL Id: jpa-00232313 https://hal.science/jpa-00232313}

Submitted on 1 Jan 1984

HAL is a multi-disciplinary open access archive for the deposit and dissemination of scientific research documents, whether they are published or not. The documents may come from teaching and research institutions in France or abroad, or from public or private research centers.
L'archive ouverte pluridisciplinaire HAL, est destinée au dépôt et à la diffusion de documents scientifiques de niveau recherche, publiés ou non, émanant des établissements d'enseignement et de recherche français ou étrangers, des laboratoires publics ou privés. 


\title{
Some possible effects modifying the far infrared synchrotron radiation spectrum
}

\author{
R. Coïsson \\ Istituto di Fisica, Università di Parma, 43100 Parma, Italy
}

(Reçu le 9 septembre 1983, accepté le 28 novembre 1983)

\begin{abstract}
Résumé. - La distribution spectrale-angulaire du rayonnement synchrotron dans l'infrarouge lointain $\left(10^{11}-10^{13} \mathrm{~Hz}\right)$ peut être modifiée par l'effet des bords des aimants, l'ouverture limitée d'obșervation et l'interférence de la lumière issue de points différents de la trajectoire, ainsi que par la superposition cohérente de la lumière produite par différents électrons du faisceau. Même si ces effets influencent plutôt la partie basse fréquence de ce spectre, et si plusieurs de ces effets sont réduits ou annulés lorsqu'on intègre sur les angles (à cause de la distribution angulaire ou de position des électrons), ils méritent cependant une étude - à la fois théorique et expérimentale approfondie.
\end{abstract}

\begin{abstract}
Effect of magnet edges, limited acceptance and interference of light coming from different points of the electron trajectory, as well as coherent addition of light from different electrons in a bunch, can modify the spectral-angular distribution of synchrotron radiation in the far infrared range $\left(10^{11}-10^{13} \mathrm{~Hz}\right)$. Although these effects might influence more likely only the lower-frequency part of this range, and many of them might be reduced or washed out by integration over angles, and by the spread in positions and angles of the electrons, they deserve a more detailed theoretical and experimental analysis.
\end{abstract}

Besides its use in the X-ray and UV regions of the spectrum, synchrotron radiation (SR) is now receiving attention as a light source also in the IR and FIR (Refs. [1-3]), because of some interesting characteristics : a higher spectral density than a black-body as $\omega \rightarrow 0$ (then in far IR), a time structure with regular short pulses, and its calculability, which suggests its use as a standard for calibrations.

As ports for FIR in SR facilities have just been set up (Ref. [3]), or will be in the near future, it might be of interest to point out possible effects influencing the FIR SR spectrum, say $10^{11}$ $10^{13} \mathrm{~Hz}$ (or 3-300 $\mathrm{cm}^{-1}$ or $3 \mathrm{~mm}-30 \mu \mathrm{m}$ ), in order to warn users, and to suggest experiments aimed at verifying the existence of these effects and allowing a quantitative analysis of them. Here we limit ourselves to a qualitative analysis and an indication of order of magnitude of some examples for realistic parameters of machine and apparatus.

In the ideal case of a single particle on a circular trajectory in free space, the e.m. field seen by the observer is qualitatively as in figure 1 , and its integral in time is zero, therefore the spectrum tends to zero as $\omega \rightarrow 0$. 


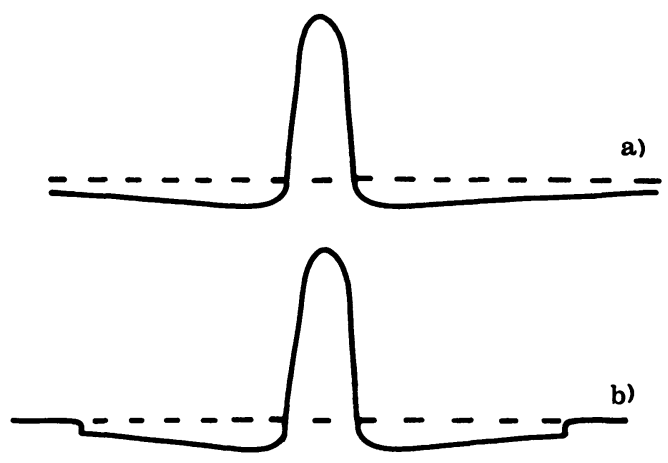

Fig. 1. - Qualitative aspect of electric field pulse in time, as seen by an observer, emitted by a single particle : a) in circular motion, b) at the centre of a magnet of limited length.

The spectrum of the radiation (power per unit solid angle and frequency) emitted by one particle at $\theta=0$ (i.e. in the plane of the orbit, of radius $R$ ), is, for $\omega \ll \omega_{c}$ where $\omega_{\mathrm{c}}=3 \gamma^{3} c / 2 R$ :

$$
\frac{\mathrm{d} W(\theta=0)}{\mathrm{d} \theta \mathrm{d} \omega}=\frac{9}{4.2^{1 / 3} \cdot \pi \cdot \Gamma^{2}(1 / 3)} \frac{W_{0}}{\omega_{\mathrm{c}}} \gamma\left(\frac{\omega}{\omega_{\mathrm{c}}}\right)^{2 / 3} \simeq 0.079 \gamma \frac{W_{0}}{\omega_{\mathrm{c}}}\left(\frac{\omega}{\omega_{\mathrm{c}}}\right)^{2 / 3}
$$

where $W_{0}$ is the total power emitted (per turn or per milliradian...), and integrating over the whole solig angle :

$$
\frac{\mathrm{d} W}{\mathrm{~d} \omega}=\frac{9}{2.2^{1 / 3} \Gamma(1 / 3)} \frac{W_{0}}{\omega_{\mathrm{c}}}\left(\frac{\omega}{\omega_{\mathrm{c}}}\right)^{1 / 3} \simeq 1.33 \frac{W}{\omega_{\mathrm{c}}}\left(\frac{\omega}{\omega_{\mathrm{c}}}\right)^{1 / 3}
$$

(and 3/4 of it is polarized in the plane of the orbit).

The spectrum of the whole beam, as long as particle times of arrival are uncorrelated (incoherent radiation) is multiplied by the number of electrons per unit time. The effects modifying this spectrum can be of various kinds :

1) The trajectory is not circular : effect of edges of magnets.

2) The observer sees each particle in different points of the trajectory because of deflection of radiated field due to reflections etc. (vacuum chamber, focussing elements, ...).

3) The observer does not see a long arc of trajectory, because of obstructions in between (limited acceptance).

4) Coherent addition of fields from different electrons in a bunch; effect possibly enchanced and extended to higher frequencies by " turbulence " in the beam. At wavelengths comparable to the bunch length, it becomes a dominant process.

5) Multiple reflections : waveguide effects, leading to suppression or enhancement of specific modes. This effect becomes a dominant process at wavelengths comparable to the vacuum chamber dimensions (several $\mathrm{cm}$ ). The effect of this on the total emitted power has been treated (Ref. [5]). Here we do not try to describe the influence of this on the FIR spectrum, then we do not consider this case.

Let us now take various cases and give examples :

1) Edge effects. If we consider a magnet of finite length $2 R \alpha$ with the tangent to the observer at its centre, the pulse seen by the observer (Fig. $1 b$ ) is " cut off " outside a time interval $2 \tau$ 
such that :

$$
2 \tau=\frac{\text { arc }}{v}-\frac{\text { chord }}{c}=\frac{2 R \alpha / c}{1-\frac{1}{2} \gamma^{2}}-\frac{2}{c} R \sin \alpha \simeq \frac{R \alpha}{c}\left(\frac{1}{\gamma^{2}}+\frac{\alpha^{3}}{3}\right) \simeq \frac{R \alpha^{3}}{3 c}
$$

so that (convolution theorem) the spectrum is convoluted by the FT of a rectangle of widths $2 \tau$. Then the cusp at $\omega=0$ (let us consider also negative frequencies) is smoothed and the spectrum is modified as in figure 2 , which has been numerically calculated for a given value of the parameters. The value at $\omega=0$ is equal to the area of the curve in figure 1 that has been cut off (Ref. [6]). In practice the situation will rather be asymmetric, with one edge not far from the observation point : this can be calculated numerically in the same way.

2) Interference of reflected light. In some cases a reflected pulse can travel in the same direction as the one directly emitted, as in the case of reflections from the vacuum chamber indicated in figure 3.

The time lag between the two pulses is :

$$
\tau=\frac{R \alpha}{v}-\frac{2 R \sin \frac{\alpha}{2}}{c}
$$

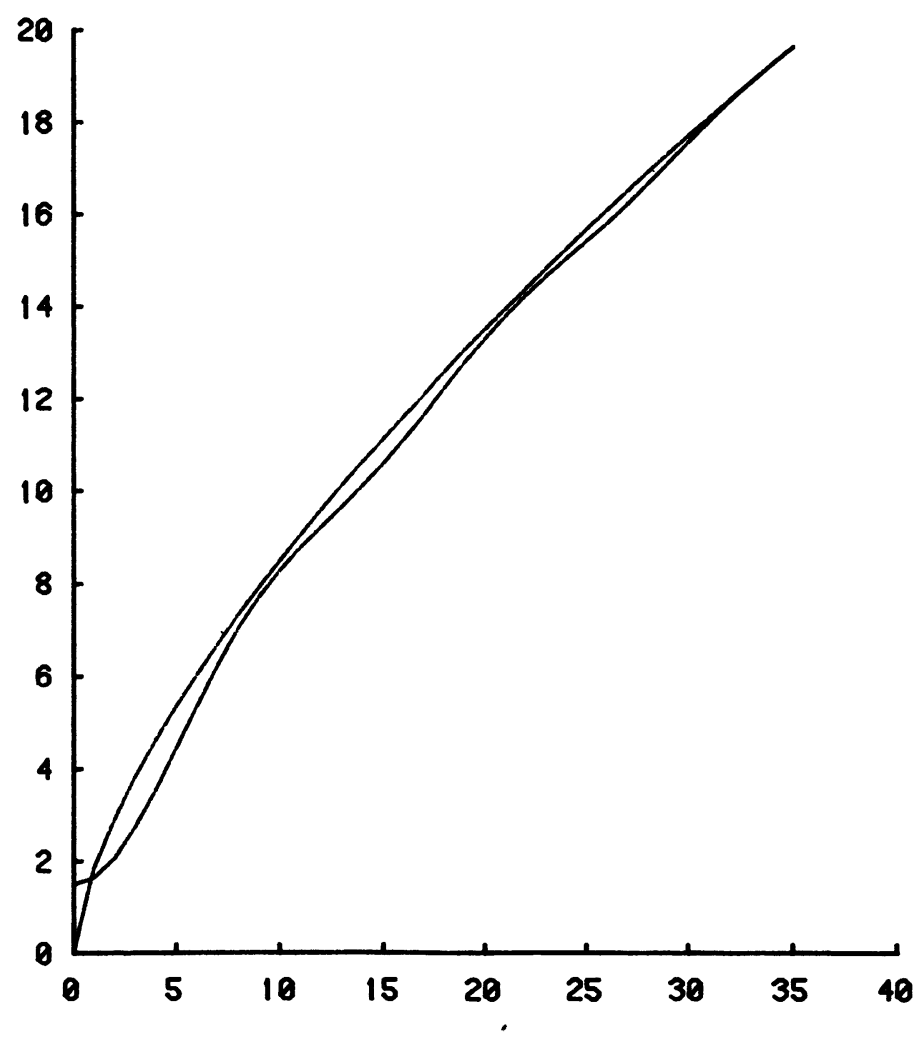

Fig. 2. - Spectrum of light from the centre of a short magnet (Fig. $1 b$ ) at $\theta=0$, numerically calculated for $R=5 \mathrm{~mm}$ and $2 R \alpha=40 \mathrm{~cm}$. Frequency is in units $2.5 \mathrm{~cm}^{-1}$, intensity in arbitrary units. Oscillations are due to interference between opposite edges of the magnet. 


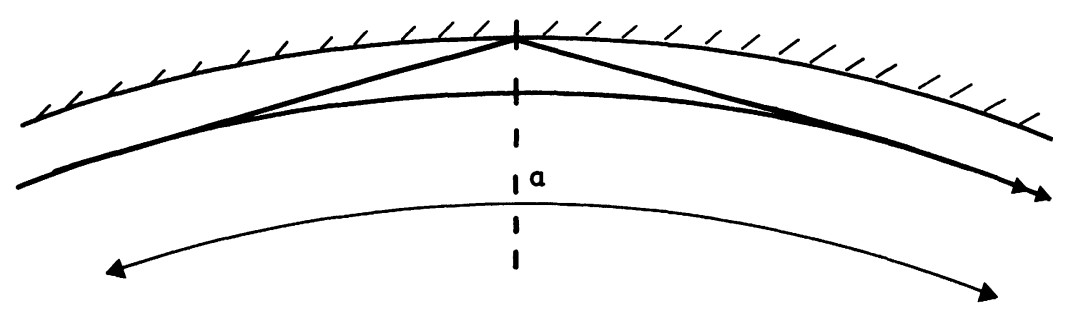

Fig. 3. - Reflection from outer wall of vacuum chamber.

as :

$$
\begin{gathered}
h=\frac{R}{\cos \frac{\alpha}{2}}-R \simeq R \alpha^{2} / 8 \\
\tau=\frac{R \alpha}{2 c}\left(1+\frac{1}{2 \gamma^{2}}\right)-\frac{R}{c}\left(\frac{\alpha}{2}-\frac{\alpha^{3}}{48}\right)=\frac{R \alpha}{2 c}\left(\frac{1}{2 \gamma^{2}}+\frac{\alpha^{2}}{24}\right) \simeq \frac{1}{3 c}\left(\frac{2 h^{3}}{R}\right)^{1 / 2} .
\end{gathered}
$$

The spectrum (at $\theta=0$ ) is in this case (analogous to the Alford-Gold experiment, Ref. [7]), multiplied (in case of two equal intensities) by a $\cos \frac{\omega \tau}{2}$ function, then has maxima for $\omega$ multiple of $2 \pi / \tau$. For $R=5 \mathrm{~m}, h=8 \mathrm{~cm}, \frac{2}{c \tau}=4 \mathrm{~cm}^{-1}$.

Let us now consider a mirror, focussing an arc of trajectory onto one point (for ex. the entrance slit of a spectrometer). The time delay between arrivals at $\mathbf{S}$ of the pulses emitted at $\mathbf{A}$ and $\mathbf{B}$ is :

$$
\tau=\frac{R \alpha}{c}\left(\frac{1}{2 \gamma^{2}}+\frac{\alpha^{2}}{24}\right) \simeq \frac{R \alpha}{c} \frac{\alpha^{2}}{24} .
$$

The amplitude, as a function of time, of the light passing through a narrow slit at $\mathbf{S}$ is then a convolution of the usual pulse (Fig. 1a) with a rectangle function of width $\tau$. Qualitatively, this looks as in figure 4.

This is only for a narrow slit, of width $<\lambda / \psi$ ( = separation of fringes produced by light at wavelength $\lambda$ at an angle $\psi$ ). Moreover, for the whole beam, the spread in electron angles and in observation angles tends to blur the interference effects, just as in the case of undulators.

With special mirrors, peculiar effect could be seen : for example if the mirror is made of $N$ (for ex. $N=3$ or 4) strips, each longer than $R / \gamma$, the spectrum through a narrow slit at $\theta=0$ (if the beam width is less than $\lambda / \alpha$ ), would be a band of relative bandwidth $1 / N$, as from an undulator.

In the particular case of interferences in the direction of straight sections, they can easily be seen also in the visible, and they have been described by some authors (Ref. [8]).

3) Obstructions. With suitable screens, the time dependence of the field seen by the observer can be modified as in the preceding two cases, and the way of calculating the spectrum is the same. This way of modifying the spectrum could be useful to make experiments to verify the

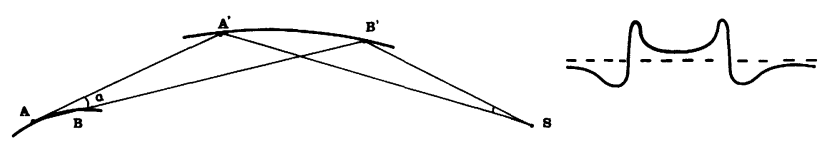

Fig. 4. - Reflection for a focussing mirror, and pulse through a narrow slit at $\mathrm{S}$. 
dependence of the spectrum from various parameters : for example with a screen before the spectrometer entrance slit, one could see only a part of the mirror, or simulate a multiple-slit mirror.

4) Coherent emission. If the longitudinal distribution of the bunch is an ideal smooth Gaussian of length $c T$, i.e. (in time) $\rho(t)=\exp \left(-\frac{1}{2} t^{2} / T^{2}\right)$, the spectrum of coherent radiation at a given angle is equal to the product of the single-particle spectrum times the FT of $\rho(t)$, i.e. $\tilde{\rho}(\omega)=$ $\exp \left(-\frac{1}{2} T^{2} \omega^{2}\right)$. Remark : while the single-particle (incoherent) spectrum is dependent on angle, the factor $\exp \left(-\frac{1}{2} T^{2} \omega^{2}\right)$ is not (the duration of the bunch does not depend on the observation angle $\theta$ ). At low frequencies, the coherent intensity tends to be $N$ times the incoherent one, where $N$ is the number of electrons in a bunch. If $N \simeq 10^{11}$ and $T \simeq 70$ ps as at Daresbury, coherent emission is appreciable $\left(N \exp \left(-\frac{1}{2} T^{2} \omega^{2}\right) \gtrsim 1\right)$ for $\lambda \gtrsim 8 \mathrm{~mm}$.

Therefore, if the bunch has the ideal equilibrium form, the effect of coherent emission in the range that we consider $(\lambda<3 \mathrm{~mm})$ is negligible (see Ref. [9]).

Coherent emission effects could anyway be observed if the longitudinal beam profile is fluctuating around the Gaussian, having as a consequence a shorter correlation length. We have such a situation in the so called" " turbulent bunch lengthening ", whose physical mechanism is like the "free electron laser" : various reflected waves propagating in the vacuum chamber, with various frequencies and phases, interact with the bunch and produce stimulated SR and a random bunch substructure. The overall process is complicated, but it is clear that a measurement of spectrum with and without turbulent bunch lengthening could give information on the spectrum of bunch profile fluctuations, which might well have appreciable components at $\lambda<1 \mathrm{~mm}$ and decrease relatively slowly with $\lambda$ (in relation with this we can remind that the ratio of coefficients of spontaneous and stimulated emission is proportional to $\omega^{3}$ ).

In conclusion, these examples suggest that there are several effects that can modify the FIR SR spectrum, although many of them will be reduced or washed out if the radiation is not selected in angle (or if position or angular spreads are too large), and the influence is likely to be effective only at the longest wavelengths (of the order of a $\mathrm{mm}$ ). These effects would depend on specific characteristics of the vacuum chamber, point of observation with respect to magnets, focussing optics, entrance slit of spectrometer, etc., then it is difficult to determine the importance of each a priori, and an experimental study is necessary, in which as many parameters as possible are varied, in order to distinguish the various effects, and to allow a further quantitative analysis. Possible experiments should include a horizontal slit in the focal plane, in order to select an angle $\theta$, and among parameters to be varied are : beam current (in order to have turbulent bunch lengthening or not), beam position, width of entrance slit of spectrometer, screens to see only a part of the mirror (for ex. to observe the beam near to or far from a magnet edge, or to apparently divide it in 2 or 3 strips, ...).

\section{Acknowledgments.}

The author acknowledges useful discussions with A. Hofmann and with M. W. Poole, and thanks A. Renieri for the connection of the turbulent bunch lengthening with the free electron laser. 
References

[1] LoPEZ-Delgado, R., Application of $S R$ to the study of relaxation processes in molecular systems in proc. of course on SR research (ICAP-INFN), directed by H. Winick, Alghero 1976, Vol. 1, ed. A. N. Mancini, I. F. Quercia and S. Stipchich.

[2] MeYer, P. and Lagarde, P., «SR in the IR », J. Physique 37 (1976) 1387.

[3] Duncan, W. D. and YARWOod, J., First detection of FIR photons from a SR source, tech. memo. DL/ SCI/TM32E (Daresbury Laboratory 1982), and summary in " SR " appendix to the Daresbury annual report $1981 / 82$.

[4] See JACKsON, J. D., Classical electrodynamics (Wiley, N. Y.) 1962, and LANDAU, L. and LIFSHITS, I., Théorie des champs (MIR, Moscow) 1960.

[5] SCHIFF, L. I., Production of particle energies beyond $200 \mathrm{MeV}$, Rev. Sci. Instrum. 17 (1946) 6.

[6] Bessonov, E. G., On a class of e.m. waves, Zh. Eksp. Teor. Fiz. 80 (1981) 852 (English transl. : Sov. Phys. JETP 53 433).

[7] Alford, W. P. and Gold, A., Laboratory measurement of the velocity of light, Amer. J. Phys. 26 (1958) 481.

[8] Nikitin, M. M., Medvedev, A. F., MoisseYev, M. B. and Epp, V. Ya., Zh. Eksp. Teor. Fiz. 79 (1980) 163 ;

Alferov, D. F. and Bashmakov, Yu. A., Pis'ma JETP 34 (1981) 15 ;

Alekseyev, V. I., Bessonov, E. G., Kahinin, A. V. and Krasikov, V. A., Preprint FIAN 228, Moscow 1981.

[9] CurTis Michel, F., Intense submillimeter radiation in electron storage rings, Phys. Rev. Lett. 48 (1982) 580. The semi-quantitative argument used in this paper to evaluate the spectrum is equivalent to assuming a square longitudinal density distribution, which is not realistic, and the result can differ by several orders of magnitude from that for a Gaussian bunch. 